\title{
Generic Small Scale Channel Model for On-Body UWB WBAN Communications
}

\author{
Timo Kumpuniemi, Matti Hämäläinen, Tommi Tuovinen, Kamya Yekeh Yazdandoost, Jari linatti \\ Centre for Wireless Communications \\ P.O. Box 4500 \\ FI-90014 University of Oulu, FINLAND \\ + 358294482882 \\ forename.surname@ee.oulu.fi
}

\begin{abstract}
This paper presents a generic small scale channel model for ultra wideband wireless body area network communications. It is based on static on-body measurements in an anechoic chamber by using a vector network analyzer within a 2-8 GHz bandwidth. Two antenna types are used for the examination: dipole and double loop. From the existing data, a generic average channel impulse response (CIR) was extracted resulting 11 and 12 resolvable paths for the dipole and the double loop antenna, respectively, when a dynamic range of $25 \mathrm{~dB}$ was used. The CIR envelopes were modelled by using the polynomial least squares (LS) fitting with orders one to five. The $5^{\text {th }}$ order LS model was noted to follow the CIR envelope most precisely. The CIR decays slightly faster for the dipole antenna. The statistical properties of the CIR bins were solved by fitting the data for 17 continuous distributions and ranking them by using the second order Akaike information criterion. To model the CIR amplitudes exactly, four different distributions were needed for the dipole but for an approximate model Weibull and lognormal distributions suffice. For the double loop, all CIR bins follow the inverse Gaussian distribution. The distributions of the CIR bin indexes were found to follow the negative binomial distribution for both antennas.
\end{abstract}

\section{Categories and Subject Descriptors}

J.2 [Physical Sciences and Engineering]: - electronics, engineering, physics.

\section{General Terms}

Measurement, Experimentation.

\section{Keywords}

Body area network, small scale channel model, ultra wideband.

\section{INTRODUCTION}

The rising life expectancies and middle ages of population in many nations worldwide [1] have partly caused increasing interest in various kinds of activities in the field of medical information and communication technology (ICT). With the help of ICT, it is

Permission to make digital or hard copies of all or part of this work for personal or classroom use is granted without fee provided that copies are not made or distributed for profit or commercial advantage and that copies bear this notice and the full citation on the first page. To copy otherwise, to republish, to post on servers or to redistribute to lists, requires prior specific permission and/or a fee.

UWBAN-2013 2013, September 30-October 02

Copyright $\odot 2013$ ICST 978-1-936968-89-3

DOI 10.4108/icst.bodynets.2013.253673 possible to improve the quality of life and treatment of people, since, e.g., a patient's vital functions can be monitored remotely to make the work of the medical staff more efficient or to enable many patients to live longer in their homes [2]. The feature of wireless technology gives more freedom in movements both at home and hospitals. The development in miniaturization of electric devices eases up their usage closer and closer to human body (on-body) and even inside them (in-body) as implants. With ultra wideband (UWB) wireless body area network (WBAN) communications, the goals are achievable and therefore the activities within the UWB WBAN have been vivid for some time $[3,4]$. UWB is a highly suitable technique for short range communications, as in WBANs [3]. The Institute of Electrical and Electronics Engineers (IEEE) released a standard referred as IEEE 802.15.6 in 2012 containing definitions for WBAN [5]. According to it, UWB technology is to be used within the frequency ranges of 3.2448-4.7424 GHz (low band) and 6.24$10.2336 \mathrm{GHz}$ (high band). It is highly realistic to expect that the published standard will increase the interest both in academia and industry on the UWB WBAN technologies.

This paper presents a small scale UWB WBAN channel model. Since the links are not divided into categories before the analysis, a generic on-body modelling is in question. The work is based on frequency domain measurements with a vector network analyzer (VNA) across the 2-8 GHz bandwidth (BW). The static measurement campaign is conducted in an anechoic chamber by using two different planar UWB antennas: dipole and double loop. The measured data is recorded and post-processed to extract the time domain parameters for the model. One motivation is to examine the effect of the antenna type on the generic UWB onbody channel model. The work is a part of a larger WBAN research on channel measurements and characterization The paper continues the work introduced in [4].

\section{MEASUREMENT ARRANGEMENT}

The anechoic chamber had a floor size of $245 \mathrm{~cm}$ by $365 \mathrm{~cm}$ and a height of $240 \mathrm{~cm}$. The $183 \mathrm{~cm}$ tall male test person was wearing a cotton T-shirt and jeans but no shoes. All possible metal containing parts such as watch, belt, ring etc. were absent during the measurements to reduce the reflections.

The VNA was a four-port device ZVA8 manufactured by Rohde \& Schwartz. Eight meters long Huber + Suhner SUCOFLEX 104PEA measurement cables were connected to each port. A computer with the LabVIEW software was used to control the VNA through the general purpose interface bus (GPIB). During the measurements, 100 consecutive frequency sweeps having 1601 frequency points in one sweep were recorded for each link. 
Both forward and reverse links were measured. The power at the transmit port was $+10 \mathrm{dBm}$. Additional information of the measurement setup is available in [4]. The structures together with the simulated and measured performances of the UWB antennas used in this work are described in detail in [6-7].

\section{IMPLEMENTED MEASUREMENTS}

At first, 14 locations to place the antennas were selected, as shown in Fig. 1. Further, six combinations of four locations were defined, since four antennas were possible to be mounted simultaneously. The chosen compositions are listed in Table 1. As noted, all options contain the point AP [4].

The point AP was situated either as an on-body measurement spot on the abdomen as shown in Fig. 1 or as an off-body spot on a pole. Since only the on-body cases are considered in this paper, the off-body channels were omitted from the data processing. All compositions were measured by using four similar prototype antennas. The work was repeated for both antenna types. A 20 $\mathrm{mm}$ thick piece made of ROHACELL $31 \mathrm{HF}$ [8] with electrical properties similar to air $\left(\varepsilon_{\mathrm{r}}=1.05 @ 2.5 \mathrm{GHz}, 1.043 @ 5 \mathrm{GHz}\right.$, $1.046 @ 10 \mathrm{GHz}$ ) was inserted between the antennas and body to maintain the antenna-body distance constant. The antennas were attached in their sites with elastic bands and painter's masking tape. Finally, the entire measurement program was repeated as such in order to diminish the effect of the variation in the antenna placements on the results [4]. In total, 252 links were measured.

\section{OBTAINED RESULTS}

\subsection{Data Post-processing Method}

The recorded data was post-processed with the MATLAB software. The frequency domain channel responses corresponding to the scattering parameter $S_{21}$ were transformed into time domain by using the complex baseband inverse fast Fourier transform (IFFT) method [9]. In practice, the IFFT was directly applied to the complex frequency domain data and a complex channel impulse response (CIR) was obtained. No windowing was applied. The time resolution obtained is the inverse of the $6 \mathrm{GHz}$ measurement BW, i.e., $\Delta t=\left(1 /\left(6 \cdot 10^{9}\right)\right) \mathrm{ns}=0.167 \mathrm{~ns}$. Assuming the signal propagation at the speed of the light, it corresponds to a resolution of $5 \mathrm{~cm}$ in distance. The absolute values of all CIRs (all sweeps of all links) were aligned in time, i.e., the first arriving signal path for all responses was set to be at the time instant $t=0$.

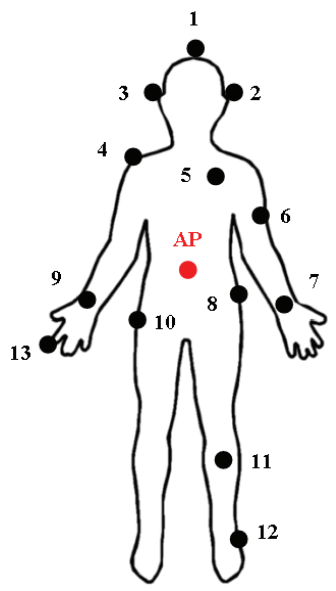

Figure 1. The selected locations for the measurements.
Table 1. The combinations of measurement locations

\begin{tabular}{|c|c|}
\hline $\mathrm{AP}-1-2-3$ & $\mathrm{AP}-10-11-12$ \\
\hline $\mathrm{AP}-5-6-7$ & $\mathrm{AP}-4-9-13$ \\
\hline $\mathrm{AP}-1-7-9$ & $\mathrm{AP}-4-8-10$ \\
\hline
\end{tabular}

\subsection{Amplitude Analysis}

\subsubsection{CIR Envelope Model}

First, the available 25200 CIRs per antenna were averaged to solve the average generic CIR envelopes for the antennas and the results were transformed into a decibel scale. The dynamic range of the generic CIRs was selected to $25 \mathrm{~dB}$, i.e., CIR taps $25 \mathrm{~dB}$ below the highest tap strength were omitted based on the following reasoning. The IEEE 802.15.6 defines the receiver sensitivity for an impulse radio UWB system with on-off keying in an additive Gaussian noise channel to be $P_{\mathrm{S}}=-76 \ldots-91 \mathrm{dBm}$ depending on the data rate used. The channel $\mathrm{BW}$ is regulated to be 499.2 MHz [5]. The Federal Communications Commission (FCC) sets the maximum transmit power in a $3.1-10.6 \mathrm{GHz}$ band to be $-41.3 \mathrm{dBm} / \mathrm{MHz}$ [10]. This corresponds to the maximum transmit power of $-14.3 \mathrm{dBm} @ 499.2 \mathrm{MHz}$ BW. The maximum values of the average CIRs are found to be $M V_{\mathrm{d}}=-57.6 \mathrm{~dB}$ (dipole) and $M V_{1}=-58.9 \mathrm{~dB}$ (double loop) corresponding the relative power of the strongest tap. With the $25 \mathrm{~dB}$ threshold used, the absolute powers of the weakest taps considered in the analysis would therefore be $-96.9 \mathrm{dBm}$ (dipole) and $-98.2 \mathrm{dBm}$ (double loop) assuming the maximum transmit power and the sensitivities above. As a result, the generic CIR for the dipole has 11 taps whereas the model for the double loop consists of 12 taps. The CIRs are not sparse in time.

Polynomial least squares (LS) data fitting was applied to the average CIRs. As a result, the CIR envelope is modelled according to the equation

$$
p(z)=p_{N} z^{N}+p_{N-1} z^{N-1}+\ldots+p_{1} z^{1}+p_{0},
$$

where $N$ is the order of the polynomial LS fit, $p_{N}$ are the model coefficients and $z \in\{0,1,2,3 \ldots\}$ is the time index. LS fitting was performed for the orders of $N=1,2,3,4$ and 5. An example of the LS fits for the dipole antenna and for the orders of 1,3 and 5 are shown in Fig. 2 where the horizontal axis contains the time delay in nanoseconds and vertical axis the relative received power in decibels. The blue dots present the true average CIR that the polynomial curves try to model. The $5^{\text {th }}$ order polynomial seems to follow the original data points better than the lower order fit curves. The goodness of the fit was evaluated by calculating the root-mean-square (RMS) error between the true average CIR envelope and its polynomial model. The full results of the LS data fitting for different orders of the fit are visible in Table 2 . The results show the general trend where the RMS error decreases as the order of the fit is raised into more complex polynomial models. For the dipole, RMS value remains almost the same at the orders of 2 and 3. For the double loop, the model orders $N=2,3$ and 4 give almost the same goodness in RMS. It should be noted that the model in (1) and Table 2 is valid for $z=0 \ldots 10$ and $z=$ $0 \ldots 11$ for the dipole and the double loop, respectively.

When examining the $p_{0}$ parameter predicting the $M V_{\mathrm{d}}$ and $M V_{1}$ values, it can be noted that the higher the fit order the better the first path is solved with the polynomial model.

For the first order model, the parameter $p_{1}$ is smaller for the dipole giving an idea that generally the CIR for the dipole decays slightly 


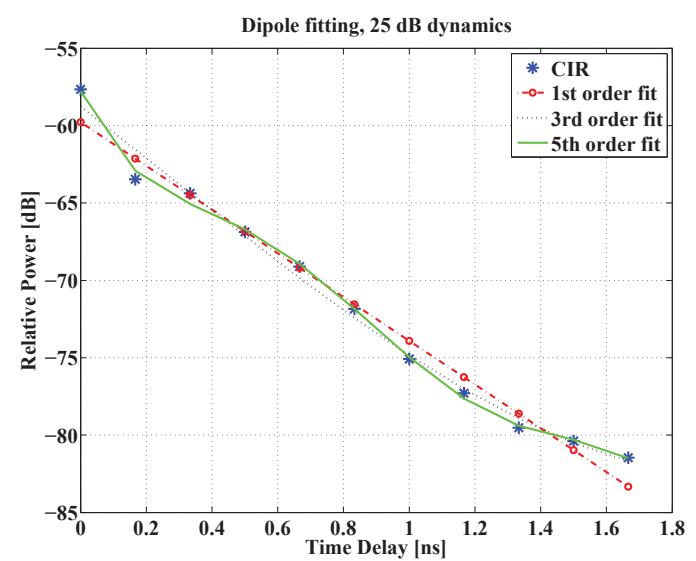

Figure 2. Dipole CIR LS fit of the order 1, 3 and 5.

Table 2. Results for $N$ th order polynomial LS data fittings

\begin{tabular}{|c|c|c|c|c|}
\hline & \multicolumn{2}{|c|}{ Dipole } & \multicolumn{2}{|c|}{ Double Loop } \\
\hline$N$ & Coefficients & RMS & Coefficients & RMS \\
\hline 1 & $\begin{array}{l}p_{1}=-2.3545 \\
p_{0}=-59.7791\end{array}$ & 1.1087 & $\begin{array}{l}p_{1}=-2.0404 \\
p_{0}=-60.7544\end{array}$ & 1.1183 \\
\hline 2 & $\begin{array}{l}p_{2}=0.0879 \\
p_{1}=-3.2332 \\
p_{0}=-58.4611\end{array}$ & 0.7918 & $\begin{array}{l}p_{2}=0.0759 \\
p_{1}=-2.8750 \\
p_{0}=-59.3634\end{array}$ & 0.7812 \\
\hline 3 & $\begin{array}{l}p_{3}=0.0086 \\
p_{2}=-0.0409 \\
p_{1}=-2.7421 \\
p_{0}=-58.7702\end{array}$ & 0.7652 & $\begin{array}{l}p_{3}=0.0028 \\
p_{2}=0.0293 \\
p_{1}=-2.6790 \\
p_{0}=-59.5031\end{array}$ & 0.7763 \\
\hline 4 & $\begin{array}{l}p_{4}=0.0082 \\
p_{3}=-0.1551 \\
p_{2}=0.9824 \\
p_{1}=-4.7888 \\
p_{0}=-58.1807\end{array}$ & 0.5784 & $\begin{array}{l}p_{4}=-0.0019 \\
p_{3}=0.0451 \\
p_{2}=-0.2617 \\
p_{1}=-2.0333 \\
p_{0}=-59.7203\end{array}$ & 0.7575 \\
\hline 5 & $\begin{array}{l}p_{5}=-0.0033 \\
p_{4}=0.0898 \\
p_{3}=-0.8676 \\
p_{2}=3.5115 \\
p_{1}=-7.8585 \\
p_{0}=-57.7891\end{array}$ & 0.3049 & $\begin{array}{l}p_{5}=-0.0023 \\
p_{4}=0.0604 \\
p_{3}=-0.5540 \\
p_{2}=2.0848 \\
p_{1}=-5.2000 \\
p_{0}=-59.2219\end{array}$ & 0.5209 \\
\hline
\end{tabular}

faster than in the case of the double loop. The number of taps is less for the dipole which also supports the same conclusion.

\subsubsection{Statistics of the Delay Bins}

The statistical characteristics of the linear scale amplitudes were extracted for each delay bin index. The measurement data was fitted for 17 theoretical continuous distributions offered by MATLAB. The tested distributions were beta (A), BirnbaumSaunders (B), exponential (C), extreme value (D), gamma (E), generalized extreme value $(\mathrm{F})$, generalized Pareto $(\mathrm{G})$, inverse
Gaussian (H), logistic (I), log-logistic (J), lognormal (K), Nakagami (L), normal (M), Rayleigh (N), Rician (O), t locationscale $(\mathrm{P})$ and Weibull $(\mathrm{Q})$ distributions [11]. The model selection problem was solved by the second order Akaike information criteria (AICc) [12]. The AICc or the classical Akaike information criteria (AIC) are used, e.g., in [13-15]. AICc is an extension to $\mathrm{AIC}$ and in large data sets, as in this paper, perform similarly to the AIC, whereas in small datasets AICc is better [12]. AIC is claimed to perform better in model selection than the methods belonging to the class of hypothesis testing [15]. Results for the distribution test for all CIR bins are shown in Table 3 in the ascending order of the models' AICc values. Thus the best distribution fit for each bin index is found as the first symbol on the left and the bin index one denoting for the first arriving path of the CIRs. For the case of the dipole antenna, it can be noted that depending on the CIR bin index the distribution that models the measurement data best is either Weibull, generalized Pareto, lognormal or log-logistic distribution. In the bins 6 and 7, the fit was not able to adapt to the Rice distribution, therefore only 16 fits are visible in these cases. The results for the double loop antennas is more straightforward, since for all bins the best fit is obtained with the inverse Gaussian distribution.

The results between the antennas in Table 3 deviate clearly, as the best distribution fits are dissimilar for all CIR bin indexes. Since the on-body antenna spots as well as the measurement setup are identical for both antennas, the explanation for the distinctions is the difference between the antenna types and the electromagnetic fields they radiate.

When examining the dipole case, it can be noted that for the third bin the difference of the AICc values between the generalized Pareto distribution $(\mathrm{G})$ and the Weibull distribution (Q) is 248 . The difference is minor compared to, e.g., the corresponding bin in the double loop case, where the difference of AICc between the distributions $\mathrm{H}$ and $\mathrm{Q}$ is 28713 . Therefore, for simplicity, the Weibull distribution can be used for the third bin also. Similarly the lognormal distribution $(\mathrm{K})$ can be used for the bins $4-11$ since the differences between the AICc values of two best fits in the 4th $(\operatorname{AICc}(\mathrm{G})-\operatorname{AICc}(\mathrm{K})=268)$ and the 7 th bin $(\operatorname{AICc}(J)-$ $\operatorname{AICc}(K)=202)$ are small among the values of all differences between consecutive AICc values. This is further verified visually in Fig. 3, where some cumulative distribution functions (CDF) are

Table 3. Results for the distribution test

\begin{tabular}{|c|l|l|}
\hline Bin & \multicolumn{1}{|c|}{ Dipole } & \multicolumn{1}{|c|}{ Double Loop } \\
\hline 1 & QGEAKCJLBFHPIMDNO & HQGEAKJBLFCPIMDNO \\
\hline 2 & QEAGKJCLFBHPIMDNO & HQEAGKJLFBCPIMDNO \\
\hline 3 & GQKJEAFBCLHPIMDNO & HQEAGKJLFBCPIMDNO \\
\hline 4 & GKJQFEACBLHPIMDNO & HKGJQFEABCLPIMDNO \\
\hline 5 & KJGFBQHEACLPIMDNO & HGQKJEAFCBLPIMDNO \\
\hline 6 & KJGFQBEAHCLPIMDN & HGKQJEAFCBLPIMNOD \\
\hline 7 & JKFGBQHEACLPIMDN & HGQKJEACFBLPIMNOD \\
\hline 8 & KJGFBQHEACLPIMDNO & HGQKEACBJFLPIMNOD \\
\hline 9 & KJFGBQHEACLPIMDNO & HQGEAKBCJLFPIMNOD \\
\hline 10 & KJGFQBEAHCLPIMDNO & HKGJBFQEACLPIMDNO \\
\hline 11 & KJGFQBEAHCLPIMDNO & HKGBJQEAFCLPIMDNO \\
\hline 12 & - & HKGBQJEAFCLPIMDNO \\
\hline
\end{tabular}




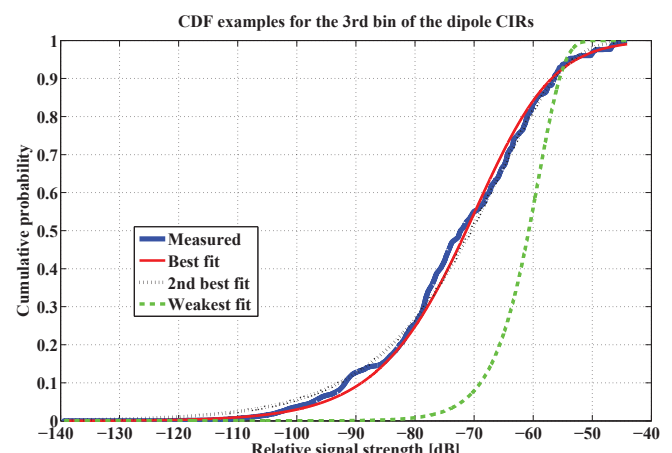

Figure 3. Exemplary CDFs for the dipole CIRs.

shown for the third dipole CIR bin. The results are given for the best and second best fits. As a reference, also the CDF giving the weakest fit is shown.

The parameters for the distributions for both antennas are listed in Table 4. The probability density functions (PDF) of the distributions are [11]

$$
f(x \mid a, b)=\frac{b}{a}\left(\frac{x}{a}\right)^{b-1} \exp \left\{-\left(\frac{x}{a}\right)^{b}\right\}, \quad x>0,
$$

for the Weibull distribution, where $a>0$ and $b>0$ are the scale and shape parameters,

$$
f(x \mid \mu, \sigma)=\frac{1}{x \sigma \sqrt{2 \pi}} \exp \left\{\frac{-(\ln x-\mu)^{2}}{2 \sigma^{2}}\right\}, \quad x>0,
$$

for the lognormal distribution with $\mu$ (real number) and $\sigma>0$ describing the mean and standard deviation of the normally distributed random variable $\ln (x)$. The inverse Gaussian PDF is described by [11]

$$
f(x \mid \alpha, \lambda)=\sqrt{\frac{\lambda}{2 \pi x^{3}}} \exp \left\{-\frac{\lambda}{2 \alpha^{2} x}(x-\alpha)^{2}\right\}, \quad x>0,
$$

where $\alpha>0$ and $\lambda>0$ are the mean and shape parameters.

Table 4. Distribution parameters for the best fits

\begin{tabular}{|c|l|l|}
\hline Bin & \multicolumn{1}{|c|}{ Dipole } & \multicolumn{1}{|c|}{ Double Loop } \\
\hline 1 & $a=0.0012, b=0.8147$ & $\alpha=0.0011, \lambda=1.0413 \cdot 10^{-4}$ \\
\hline 2 & $a=5.7829 \cdot 10^{-4}, b=0.7795$ & $\alpha=6.3532 \cdot 10^{-4}, \lambda=3.5521 \cdot 10^{-5}$ \\
\hline 3 & $a=4.8969 \cdot 10^{-4}, b=0.7345$ & $\alpha=6.4157 \cdot 10^{-4}, \lambda=4.5918 \cdot 10^{-5}$ \\
\hline 4 & $\mu=-8.6100, \sigma=1.4787$ & $\alpha=4.8655 \cdot 10^{-4}, \lambda=5.0342 \cdot 10^{-5}$ \\
\hline 5 & $\mu=-8.9040, \sigma=1.4142$ & $\alpha=3.3322 \cdot 10^{-4}, \lambda=3.1961 \cdot 10^{-5}$ \\
\hline 6 & $\mu=-9.2348, \sigma=1.4082$ & $\alpha=2.4969 \cdot 10^{-4}, \lambda=3.7238 \cdot 10^{-5}$ \\
\hline 7 & $\mu=-9.4845, \sigma=1.2954$ & $\alpha=1.9503 \cdot 10^{-4}, \lambda=4.0707 \cdot 10^{-5}$ \\
\hline 8 & $\mu=-9.7374, \sigma=1.3346$ & $\alpha=1.4940 \cdot 10^{-4}, \lambda=3.7844 \cdot 10^{-5}$ \\
\hline 9 & $\mu=-9.9844, \sigma=1.2842$ & $\alpha=1.2693 \cdot 10^{-4}, \lambda=2.5836 \cdot 10^{-5}$ \\
\hline 10 & $\mu=-10.1519, \sigma=1.3819$ & $\alpha=1.2102 \cdot 10^{-4}, \lambda=2.2066 \cdot 10^{-5}$ \\
\hline 11 & $\mu=-10.3447, \sigma=1.4312$ & $\alpha=1.0046 \cdot 10^{-4}, \lambda=1.7603 \cdot 10^{-5}$ \\
\hline 12 & - & $\alpha=7.7384 \cdot 10^{-5}, \lambda=1.5076 \cdot 10^{-5}$ \\
\hline
\end{tabular}

\subsubsection{Delay Domain Analysis of the CIRs}

The delay properties of the CIRs were also analyzed. The bins containing signal energy for the dynamic range of $25 \mathrm{~dB}$ were extracted. As a result, mean, median and maximum values for the observed bin indexes were determined and they are shown in Table 5. The bin indexes were fitted with three discrete distributions available in MATLAB with the names of binomial, negative binomial and Poisson distribution. The model that produced the smallest AICc value was the negative binomial distribution with the PDF [11]

$$
f(x \mid r, w)= \begin{cases}\left(\begin{array}{c}
r+x-1 \\
x
\end{array}\right) w^{r}(1-w)^{x}, & r \in Z \\
\frac{\Gamma(r+x)}{\Gamma(r) \Gamma(x+1)} w^{r}(1-w)^{x}, & r \in R,\end{cases}
$$

where $r$ and $0<w \leq 1$ are the parameters of the distribution. If $r$ is an integer $(Z)$, the upper equation is valid but for non-integer values $($ real, $R$ ) the lower one should be applied, where $\Gamma$ denotes for the Gamma function [11]

$$
\Gamma(x)=\int_{0}^{\infty} e^{-t} t^{x-1} d t .
$$

The parameter values in (5) are listed in Table 5 as well. In Fig.4 the CDFs for the observed bin indexes and the negative binomial distribution fitting to them is shown. The mean bin index is larger for the dipole but the median values are similar showing that the majority of the indexes is less than 10. At first the mean bin index values seem to be contradictory with the result of the average CIR tap values (11 and 12), but it should be noted that the high bin indexes are close to the threshold and therefore insignificant. After averaging high bin indexes do not exceed the threshold.

Table 5. Results for delay domain analysis

\begin{tabular}{|c|c|c|}
\hline & Dipole & Double Loop \\
\hline Mean bin & 20.5 & 17.6 \\
\hline Median bin & 9 & 9 \\
\hline Maximum bin & 97 & 102 \\
\hline$r$ & 0.6937 & 0.8021 \\
\hline$w$ & 0.0327 & 0.0436 \\
\hline
\end{tabular}
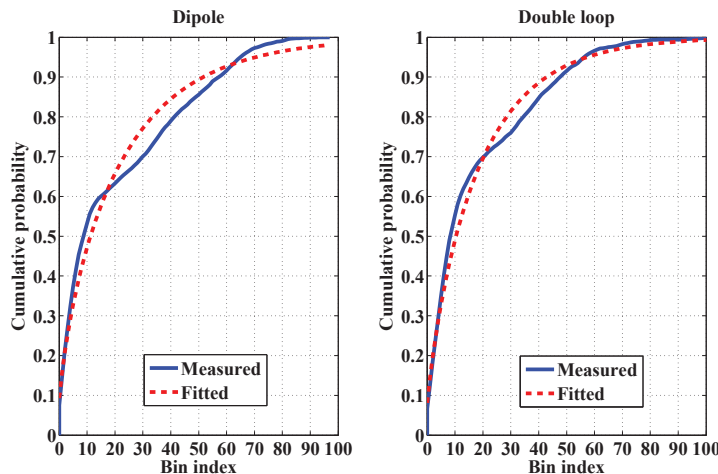

Figure 4. CIR bin CDFs and their fittings. 


\section{CONCLUSIONS AND FUTURE WORK}

A generic small scale channel modelling work was performed for UWB on-body communications based on frequency domain measurements in an anechoic chamber. Two antenna types were used: dipole and double loop. The average CIR was extracted and a channel tap number of 11 for the dipole and 12 for the double loop with a dynamic range of $25 \mathrm{~dB}$ were obtained. CIR envelope models were developed by using the polynomial LS fitting with orders one to five. The $5^{\text {th }}$ order LS model was noted to follow the CIR envelope most precisely. The CIR decays slightly faster for the dipole antenna. The distributions of the amplitudes of the CIR bins were solved by testing the measurement data against 17 continuous distributions and selecting the best candidate based on AICc. For dipoles, the exact modelling requires the usage of four different distributions, but can be approximated by Weibull and lognormal distributions. For the double loop, all CIR bins can be modelled with the inverse Gaussian distribution. The differences between the results are explained by the discrepancies of the antenna types and the electromagnetic fields they radiate.

The delay characteristics of the CIRs were also examined. The PDFs of the bin indexes within the selected $25 \mathrm{~dB}$ dynamic range were noted to follow the negative binomial distribution. Most of the bin indexes were below 10 based on their median value.

Examination of the channel models for the cases when the measured links are classified into different categories, e.g., lineof-sight and non-line-of-sight links, will be one of the topics of the future work. Also the effect of different model selection criteria on the statistical distributions beyond the used AICc will be an interesting theme to examine. Extending the investigations into the creeping waves, echoic environments, as well as pseudodynamic or even dynamic channel cases, seem also to have an outmost importance in order to explore the complex on-body UWB radio channel phenomena in a greater depth.

\section{ACKNOWLEDGMENTS}

The work has been funded by the Finnish Funding Agency for Technology and Innovation (Tekes) by the project Wireless Body Area Network for Health and Medical Care (WiBAN-HAM).

The authors wish to thank Mr. Veikko Hovinen and Mr. Risto Vuohtoniemi from Centre for Wireless Communications for fruitful discussions.

\section{REFERENCES}

[1] United Nations, Department of Economic and Social Affairs. Online, accessed in June 2013. DOI=

http://esa.un.org/unpd/wpp/population-pyramids/populationpyramids_absolute.htm.

[2] Hämäläinen, M., and Kohno, R. 2007. Prospects for wireless technology in remote care processes. In Proceedings of the 2nd International Symposium on Medical Information and Communication Technology (Oulu, Finland, Dec. 11-13, 2007). ISMICT 2007. 1-4.

[3] Hall, P. S., and Hao, Y. 2012. Antennas and Propagation for Body-Centric Wireless Communications, 2nd ed. Artech House, Norwood, MA, USA.

[4] Kumpuniemi, T., Tuovinen, T., Hämäläinen, M., Yekeh Yazdandoost, K., Vuohtoniemi, R., and Iinatti, J. 2013.
Measurement-Based On-Body Path Loss Modelling for UWB WBAN Communications. In Proceedings of the $7^{\text {th }}$ International Symposium on Medical Information and Communication Technology (Tokyo, Japan, March 6-8, 2013). ISMICT 2013. IEEE, 233-237.

[5] IEEE standard for local and metropolitan area networks, IEEE 802.15.6-2012 - Part 15.6: Wireless Body Area Networks. 2012.

[6] Tuovinen,T., Kumpuniemi, T., Yekeh Yazdandoost, K., Hämäläinen, M., and Iinatti, J. 2013. Effect of the antennahuman body distance on the antenna matching in UWB WBAN applications. In Proceedings of the $7^{\text {th }}$ International Symposium on Medical Information and Communication Technology (Tokyo, Japan, March 6-8, 2013). ISMICT 2013. IEEE 193-197.

[7] Tuovinen, T., Kumpuniemi, T., Hämäläinen, M., Yekeh Yazdandoost, K., and Iinatti, J. 2013. Effect of the antennabody distance on the on-ext and on-on channel link path gain in UWB WBAN applications. 35th Annual International Conference of the IEEE Engineering in Medicine and Biology Society (Osaka, Japan, July 3-7 2013). EMBC 2013. IEEE 1242-1245.

[8] Rohacell. Online, accessed in June 2013. DOI= http://www.rohacell.com/product/rohacell/en/productsservices/rohacell-hf/pages/default.aspx.

[9] Denis, B., and Keignart, J. K. 2003. Post-processing framework for enhanced UWB channel modeling from bandlimited measurements. In Proceedings of the 2003 IEEE Conference on Ultra Wideband Systems and Technologies (Reston, USA, Nov. 16-19 2003). UWBST 2003. IEEE 260264.

[10] Allen, B., Dohler, M., Okon, E. E., Malik, W. Q., Brown, A. K., and Edwards D.J. (editors). 2007. Ultra-wideband Antennas and Propagation for Communications, Radar and Imaging. John Wiley \& Sons, Chichester, West Sussex, England.

[11] Mathworks, MATLAB, Documentation Center, Statistics Toolbox. Online, accessed in June 2013. DOI= http://www.mathworks.se/help/stats/.

[12] Burnham, K. P., and Anderson, D. R. 2002. Model Selection and Multimodel Inference, A Practical InformationTheoretic Approach, Second Edition. Springer-Verlag, New York, USA.

[13] Schuster, U. G., and Bölcskei, H. 2007. Ultrawideband channel modeling on the basis of information-theoretic criteria. IEEE Trans. on Wireless Comm. 6, 7 (July2007), 2464-2475.

[14] Fort, A. 2007. Body Area Communications: Channel Characterization and Ultra-Wideband System-Level Approach for Low Power. Doctoral Thesis. Vrije Universiteit Brussel (VUB).

[15] Taparugssanagorn, A., Pomalaza-Ráez, C., Isola, A., Tesi, R., Hämäläinen, M., and Iinatti, J. 2010. UWB channel modelling for wireless body area networks in a hospital. Int. J. Ultra Wideband Communications and systems, 1, 4 (August 2010), 226-236. 